\begin{tabular}{c} 
Volume and Issues Obtainable at Center for Sustainability Research and Consultancy \\
Journal of Business and Social Review in Emerging Economies \\
ISSN: 2519-089X (E): 2519-0326 \\
Volume 6: Issue 2 June 2020 \\
CSRC \\
Journal homepage: www.publishing.globalcsrc.org/jbsee \\
\hline
\end{tabular}

\title{
Vacillating Behavior of TOM Effect and Adaptive Market Hypothesis: A Firm Level Evidence from Emerging Stock Market of Pakistan
}

\author{
${ }^{1}$ Dr. Muhammad Naeem Shahid ${ }^{2}$ Dr. Khalid Latif, ${ }^{3}$ Dr. Ghulam Mujtaba Chaudhary, ${ }^{4}$ Dr.Rehana Kouser \\ ${ }^{1}$ Assistant Professor, Chinot Sub Campus, Government College University, Faisalabad Pakistan, \\ naeemtuf@yahoo.com \\ ${ }^{2}$ Assistant Professor, College of Commerce, Government College University Faisalabad Pakistan, \\ khalidlatif@gcuf.edu.pk \\ ${ }^{3}$ Assistant Professor, Department of Business Administration, University of Kotli Azad Jammu and Kashmir, \\ ghulam.mujtaba@uokajk.edu.pk \\ ${ }^{4}$ Professor, Department of Commerce, Bahauddin Zakariya University Multan Pakistan. \\ rehanakouser@bzu.edu.pk
}

\begin{tabular}{l}
\hline ARTICLE DETAILS \\
\hline History \\
Revised format: May 2020 \\
Available Online: June 2020 \\
\\
\hline Keywords \\
Efficient Market Hypothesis, \\
TOM effect, Adaptive Market \\
Hypothesis
\end{tabular}

JEL Classification

M1, M2

\begin{abstract}
Through the current study we amplify the available literature on AMH (Adaptive Market Hypothesis) and calendar anomalies because this is the first study of its nature which links TOM effect with AMH which allows the behavior of conventional TOM-effect to swing over time. To fulfill the drive, study investigates daily mean return from PSX of Pakistan using data of 107 firms individually over a longer period of time ranging 1996-2015. To discover the time variation in the levels of predictability of TOM returns, study uses four different sub-samples covering identical length of observations of five years each to investigate how TOM effect has performed over time. There are few studies in the literature investigating TOM effect at firm level and very rare studies examining TOM effect through (AMH), so the current study may be of importance and interest to finance researcher, academicians and practitioners alike. To elucidate the volatility and its varying nature, the study applies $\operatorname{GARCH}(1,1)$ regression model which enables for time-variation in volatility of security returns. Kruskal-Wallis test-statistic is used to handle non normality in the equity return series. We find that with the passage of time performance of TOM effect evolves, consistent and aligned with the assertion of AMH. Finally, this study exhibits that behavior of TOM effect is well elucidated by Adaptive Market Hypothesis (AMH) than conventional Efficient Market Hypothesis (EMH). The results may be used for better decision making for investors and the article complements studies on market efficiency and TOM effect in developing and developed countries.
\end{abstract}

\section{OPEN ACCESS}

(C) 2020 The authors, under a Creative Commons Attribution-

NonCommercial 4.0

Corresponding author's email address: rehanakouser@bzu.edu.pk

Recommended citation: Shahid, M.N., Latif, K., Chaudhary, G.M., Kouser, R. (2020). Vacillating Behavior of TOM Effect and Adaptive Market Hypothesis: A Firm Level Evidence from Emerging Stock Market of Pakistan. Journal of 
Business and Social Review in Emerging Economies, 6 (2), 517-529.

\section{Introduction}

Through investigation of capricious levels of renowned turn of the month (TOM), the article tries to enhance prevailing literature on Adaptive Market Hypothesis (AMH) proposed by (Lo, 2004). TOM effect/anomaly is referred to a time period "starts from the last trading-day of the current month to the first three trading-days of the next month". The anomaly produces nosily high average returns around the TOM in stock exchanges which cast serious ambiguity on the validation of market efficiency. The notion of market efficiency and Efficient Market Hypothesis $(\mathrm{EMH})$ depicts independence of stock prices under the umbrella of weak form efficiency hence returns are unforeseeable (Fama, 1970). When the prices of stocks are dependent, the investors can use the historical information of past trading to predict and gain abnormal returns. According to (Halari, 2013; Hashmi, 2014; Shahid \& Mehmood, 2015), stock returns display serial correlation and profitable opportunities do exist in the market which contradicts EMH. Hence TOM effect prevails in the markets especially in emerging stock markets. Absolute perfect efficiency is not possible for a stock market. If market is perfectly efficient there would be no profitable opportunities exist in the market, threfore, market participants will have no advantage to collect costly information (Grossman \& Stiglitz, 1980). By keeping in view the argument of (Grossman \& Stiglitz, 1980) of "impossibility of perfectly efficient market", Campbell et al. (1997) proposes the idea of "relative efficiency" rather "perfect efficiency" which encourages the researchers to measure market efficiency from an all-or-nothing stance to measure levels of market efficiency (Shahid \& Sattar (2017).

In Pakistan, researchers (Abdul, Hung-Chun, \& Fakhar-un-Nisa, 2011; Sania \& Rizwan, 2014) in their latest studies render proof about inefficiency of Pakistani stock markets. Whereas other studies elucidate efficiency of Pakistani equity markets. Weak form efficiency of PSX is investigated by (Nisar \& Hanif, 2012) where they support weak form efficiency through monthly return data. In similar fashion Rabbani et al. (2013) conclude PSX as weak form efficient during 1999-2001 and 2005-2007. Whereas (Riaz, Hassan, \& Nadim, n.d) suggest the market efficiency alters with application of different tests that means efficiency of market may changes occasionally. Therefore, about efficiency and inefficiency of markets conflicting views have been presented. Exploration of efficiency of stock market using AMH is necessary which defines that efficiency (return predictability) alters over time. A new model is proposed by Lo (2004) namely "Adaptive Market Hypothesis (AMH)" to include varying degree of return predictability. It ease market anomalies to coexist with market efficiency. AMH facilitates efficiency of markets to evolve over time. According to AMH model, market efficiency is an "ever changing phenomenon" based upon conditions of environment and players of market. In addition, AMH illustrates market efficiency is not a warranted outcome as arbitrage opportunities for gaining extra ordinary profit is occasionally. According to Urquhart (2013), early studies are evident of market inefficiency and efficiency based on pre-determined time frames while trading environment in the market may variates over time producing fluctuations in efficiency over time which is consistence with AMH (Shahid \& Sattar, 2017; Shahid et al., 2018). The paper examines whether AMH is better explanation of behavior of TOM effect comparing to conventional EMH at firm level in Paistan as currently AMH is drawing more attention. Moreover, individual investors and security organizations may be benefitted for even better forecasting and clear understanding of market by the conclusions of the study. From January 1996 to December 2015, individual firms trading on PSX have been chosen. For subsample analysis, a fixed five-year length of sample is used to analyze TOM effect behavior.

The paper will result in enhancement of available literature on AMH and calendar anomaly (TOM). Exploration of changing behavior of TOM effect is leveraged by sub-sample analysis on the whole period of study. However, according to (Urquhart \& Hudson, 2013; Shahid \& Sattar 2017), selection of sub-samples and range of size are subjective in nature. To study how TOM effect will perform overtime, the data is split into four subsamples with same length of 5 year. Sub-samples render reasonable observations to yield authentic results that allow thorough examination of varying degree of TOM effect. So we enrich literature on AMH by satisfying the broken thread of varying degree of TOM effect by AMH in vide infra ways: Firstly, it is the first study which explores abnormality of TOM effect with AMH that allows behavior of returns regarding the TOM effect to vary over the time. Secondly, this is the first study that explores the performance of TOM effect at firm level based on AMH. Finally, the paper analyses 
behavior of TOM effect by applying a GARCH $(1,1)$ regression model which eases the unstable nature of volatility in equity returns. Moreover, Kruskal-Wallis test statistic is used to leverage the non-normal behavior of stock return data. The results of this study depicts that behavior of TOM effect gradually varies with time as performance of this effect changes from time to time and persistent with AMH. The article proposes that AMH is good explanation of behavior of TOM effect comparing to traditional EMH.

\section{Literature Review}

Based on the strong agreement exists in earlier research (Lakonishok \& Smidt, 1988; McConnell \& Xu, 2008), TOM (the turn of the month) is defined as "a time period starts from the last trading-day of the current month to the first three trading-days of the next month". At the month end investors start selling of shares therefore, they look for optimistic/positive changes in upcoming month. This anomaly is defined as nosily high average returns around the TOM in the equity exchange markets. According to Camptom et al. (2006) TOM effect is the most popular and important anomaly among other kinds of calendar patterns and different researchers document this effect in national and international equity markets. In NYSE, TOM effect is first documented by Ariel (1987) over the period from 1963 to 1981, where he finds that last day of the current and first nine days of the subsequent month exhibit greater average and statistically significant returns as compared to the rest of days of month. Ariel (1987) finds $0.47 \%$ return around the turn of the month (TOM) as compared to $0.061 \%$ during any other four days period. Likewise, DJIA index is investigated by Lakonishok and Smidt (1988) over the period of years 1897-1986 and detect eight times greater average returns around TOM trading days as compared with other normal trading days of the month. This analysis of Lakonishok and Smidt (1988) is further extended by (Cadsby \& Ratner, 1992) in other countries. It is found that TOM effect is present in UK, Switzerland, Germany, Canada and Australia, while stock markets of Japan, Hong Kong, Italy and France are not evident of TOM effect. Agrawal and Tandon (1994) examine TOM effect in 18 countries from 1970-1987 and find that only 14 countries are evident of TOM-effect including most emerged exchanges of Japan (Nikkei), UK (FT30) and USA (DJIA). Hensel and Ziemba (1996) examine investment in the S\&P500-Index on TOM days and in T-bills over the other days. They report that the turn-of-the-month strategy outperformed a baseline strategy by $0.63 \%$ per year over the period 1928-1993”.

With the application of several tests, Wong at al. (2006) investigate TOM effect and find that it exhibits greater positive returns as compared to other trading-days of the month. By taking daily equity returns calendar anomalies are investigated by Silva (2010) over the period from 1989 to 2008 in Portuguese stock exchange. With the application of OLS, significant positive returns are observed on last and first 5 days of the month. Also, exchange traded funds and S\&P 500-index exhibit highest returns around TOM (Chen \& Chua, 2011). Ehsan (2012) describes that psychology of the investors directs the calendar anomalies at PSX. She finds positive and statistically significant TOM in daily returns over the period of 2002 to 2004 . But the small sample size of the study is the basic limitation of her research work. From 1991 to 1997, Zafar et al. (2012) examine the presence of TOM effect at KSE-100 index of PSX. They find the TOM anomaly in the full as well as in certain sub periods like during 1991-1993 and 2002-2005. The time variation in the behavior of TOM effect is against the assumptions of EMH and supports AMH.

\section{Data and Methodology}

In this study, we consider the daily stock returns of listed firm on PSX to investigate the effect of TOM and to gauge its influence over the time. The final sample consists of 107 listed firms for which the 20 years data from January 1996 to December 2015 is available and obtained. The individual firm data is more suitable to examine the behavior of TOM effect as compared to stock exchange indices. Thus, the analysis provides a more accurate indication of whether equity returns are useful to investors on TOM and whether this effect has a cyclical nature of efficiency.

\section{Table-1. Full sample period (1996-2015) descriptive of TOM-effect}




\begin{tabular}{|l|r|r|l|l|}
\hline & \multicolumn{1}{|c|}{ Mean } & \multicolumn{1}{c|}{ Std. } & t-statistic & W-statistic \\
TOM & 0.1134 & Deviation & & \\
Non-TOM & 0.0127 & 0.0444 & $7.185 * * *$ & $53.641 * * *$ \\
\hline
\end{tabular}

Where $* * *$ show significance level at $1 \%$ level.

The regression equation is as under:

$$
R_{t}=c+\beta D_{t}+\varepsilon_{t}, \quad t=1, \ldots, T
$$

Where R_t represents the individual stock return, D_t represents indicator of TOM effect as adopted by (Urquhart \& McGroarty, 2014; Shahid \& Sattar, 2017), while $\varepsilon_{-} \mathrm{t}$ is the error term.

To investigate the existence of TOM effects on the Pakistan Stock Exchange, instead of using simple least squares regression (OLS), this study uses the GARCH (p, q) model proposed by (Bollerslev, 1986). In our analysis, we use the GARCH $(1,1)$ regression model because the GARCH $(1,1)$ model is the suitable and simplest model for the measurement of volatility and is the commonly used and applied in the literature (Engle, 2001). GARCH (1, 1) model "enables researchers to model volatility based on past variance and error, rather than fixed through the series (Urquhart \& McGroarty, 2014)". To gauge the time variant behavior of each sample firm, in this study, we use the GARCH $(1,1)$ model as under:-

$$
h_{t}=\beta_{0}+\beta_{1} \varepsilon_{t-1}^{2}+\theta h_{t-1}
$$

For each firm's return at time $t, h \_t$ represent the conditional variance, $h \_(t-1)$ symbolizes the conditional variance of each firm's stock return at time t-1. $\beta_{-} 0, \beta_{-} 1 \& \theta$ represent the parameters of GARCH $(1,1)$ regression. The GARCH model is a suitable model and has the potential to capture the desirable characteristics of stock market returns, but it is not suitable to capture the non-normality function of yield regions. Due to this reason, we use a nonparametric Kruskal-Wallis (K.W) test to examine predominant sensitivity of population to difference in mean and whether the population has identical distributions from which the samples are drawn. Thus, we investigate the mean differences in the stock returns around TOM and on non-TOM days so that;

$$
H=\left(\frac{12}{N(N+1)} \sum_{j=1}^{k} \frac{R_{j}^{2}}{n_{j}}\right)-3(N+1)
$$

Where total number of observations is denoted by $\mathrm{N}, \mathrm{k}$ represents the number of groups, $\mathrm{n}_{-} \mathrm{j}$ shows the total observations, $R \_j^{\wedge} 2$ denotes the average rank of observations in the jth group. Therefore, to test how the TOM effect actually varied through time, we apply the Kruskl - Wallis test and the GARCH regression model in the full-sample as well as fixed-length subgroups. We divide our data into sub-samples for a period of 5 years, thus generating 4 sub-

\begin{tabular}{|c|c|c|c|c|c|c|c|c|}
\hline $\begin{array}{l}\text { TOM } \\
\text { Effect }\end{array}$ & Firms & Mean & Firms & Mean & Firms & Mean & Firms & Mean \\
\hline TOM & PK:ABB & 0.174 & PK:DEG & 0.258 & PK:JIN & 0.199 & PK:TLM & 0.232 \\
\hline Non-TOM & & 0.024 & & -0.008 & & 0.039 & & -0.067 \\
\hline TOM & PK:ADI & 0.21 & PK:ETU & 0.082 & PK:KIE & 0.28 & PK:PTC & 0.244 \\
\hline Non-TOM & & 0.009 & & 0.064 & & -0.091 & & 0.025 \\
\hline TOM & PK:AGR & 0.052 & PK:ERO & 0.279 & PK:KRM & -0.077 & PK:PSM & 0.064 \\
\hline Non-TOM & & 0.077 & & -0.003 & & 0.025 & & -0.028 \\
\hline TOM & PK:AGT & 0.171 & PK:FSM & -0.009 & PK:KWG & -0.055 & PK:LAK & 0.155 \\
\hline Non-TOM & & 0.044 & & 0.056 & & 0.053 & & 0.089 \\
\hline
\end{tabular}
examples of identical lengths to estimate the

Table-2. Full sample period (1996-2015), Mean Returns on non-TOM days and TOM days for sample firms 


\begin{tabular}{|c|c|c|c|c|c|c|c|c|}
\hline TOM & PK:ACB & 0.323 & PK:FAU & 0.271 & PK:KNR & 0.26 & PK:PCT & 0.336 \\
\hline Non-TOM & & -0.026 & & -0.004 & & -0.01 & & -0.02 \\
\hline TOM & PK:ATH & 0.047 & PK:FZM & 0.052 & PK:LDP & 0.109 & PK:POC & 0.106 \\
\hline Non-TOM & & 0.113 & & 0.023 & & -0.026 & & -0.024 \\
\hline TOM & PK:ATR & 0.15 & PK:FEC & 0.076 & PK:MLC & 0.32 & PK:RMP & 0.025 \\
\hline Non-TOM & & 0.026 & & 0.011 & & -0.036 & & 0.09 \\
\hline TOM & PK:BKP & 0.097 & PK:NAK & -0.001 & PK:MBK & 0.286 & PK:RUP & -0.02 \\
\hline Non-TOM & & 0.012 & & 0.025 & & 0.021 & & -0.026 \\
\hline TOM & PK:BAP & 0.114 & PK:GAI & 0.067 & PK:MIR & -0.066 & PK:STM & -0.018 \\
\hline Non-TOM & & 0.084 & & 0.021 & & 0.056 & & 0.001 \\
\hline TOM & PK:BHA & -0.012 & PK:GTR & 0.166 & PK:MRB & 0.064 & PK:CCB & 0.191 \\
\hline Non-TOM & & 0.05 & & 0.037 & & 0.094 & & -0.091 \\
\hline TOM & PK:BOC & 0.029 & PK:GWC & -0.084 & PK:NAR & 0.25 & PK:SAN & 0.099 \\
\hline Non-TOM & & 0.054 & & 0.021 & & -0.014 & & -0.007 \\
\hline TOM & PK:CAL & 0.424 & PK:GLT & 0.201 & PK:NPK & 0.093 & PK:HPN & 0.063 \\
\hline Non-TOM & & -0.081 & & 0.003 & & 0.092 & & 0.027 \\
\hline TOM & PK:CPB & 0 & PK:GRY & 0.057 & PK:NAT & 0.11 & PK:SPP & 0.254 \\
\hline Non-TOM & & 0.032 & & 0.019 & & -0.046 & & 0.025 \\
\hline TOM & PK:CTC & 0.277 & PK:GUL & 0.122 & PK:NHT & 0.183 & PK:SAP & 0.097 \\
\hline Non-TOM & & -0.022 & & 0.021 & & 0.03 & & 0.036 \\
\hline TOM & PK:CSA & 0.158 & PK:GSM & 0.002 & PK:NON & -0.139 & PK:SEA & 0.122 \\
\hline Non-TOM & & 0.015 & & -0.026 & & 0.074 & & 0.078 \\
\hline TOM & PK:CTX & 0.037 & PK:HAB & 0.233 & PK:ORI & 0.082 & PK:SER & 0.074 \\
\hline Non-TOM & & 0 & & -0.005 & & 0.004 & & 0.046 \\
\hline TOM & PK:CYA & 0.116 & PK:MET & 0.164 & PK:PAC & 0.176 & PK:SHA & 0.019 \\
\hline Non-TOM & & 0.049 & & 0.037 & & 0.015 & & 0.034 \\
\hline TOM & PK:DAC & 0.178 & PK:HSM & 0.264 & PK:PET & 0.172 & PK:SCM & -0.208 \\
\hline Non-TOM & & -0.072 & & -0.004 & & 0.025 & & 0.052 \\
\hline TOM & PK:DAE & 0.013 & PK:HAE & 0.206 & PK:PSM & 0.203 & PK:SHJ & 0.005 \\
\hline Non-TOM & & -0.011 & & -0.027 & & 0.038 & & 0.026 \\
\hline TOM & PK:DAN & 0.165 & PK:HPM & 0.054 & PK:PNC & -0.096 & PK:SHK & -0.067 \\
\hline Non-TOM & & -0.046 & & 0.064 & & 0.094 & & 0.033 \\
\hline TOM & PK:DDH & 0.278 & PK:HUB & 0.367 & PK:PEN & 0.014 & PK:PBS & 0.219 \\
\hline Non-TOM & & 0.016 & & -0.047 & & 0.079 & & -0.011 \\
\hline TOM & PK:DAW & 0.204 & PK:HUF & 0.16 & PK:PAL & 0.058 & PK:SIT & 0.051 \\
\hline Non-TOM & & 0.037 & & 0.029 & & -0.013 & & 0.037 \\
\hline TOM & PK:DKT & 0.06 & PK:ICI & 0.229 & PK:PNS & 0.214 & PK:SON & 0.184 \\
\hline Non-TOM & & -0.06 & & -0.027 & & 0.023 & & 0.009 \\
\hline TOM & PK:DMT & 0.094 & PK:IMO & 0.136 & PK:POF & 0.33 & PK:SNG & 0.231 \\
\hline Non-TOM & & -0.064 & & 0.066 & & 0.002 & & -0.037 \\
\hline TOM & PK:DES & 0.032 & PK:INI & 0.092 & PK:PRE & 0.185 & PK:SUI & 0.24 \\
\hline Non-TOM & & -0.071 & & 0.044 & & -0.009 & & -0.023 \\
\hline TOM & PK:DSM & -0.171 & PK:ASB & 0.051 & PK:PSO & 0.194 & PK:TRP & -0.549 \\
\hline Non-TOM & & -0.005 & & -0.048 & & -0.004 & & 0.095 \\
\hline TOM & PK:DEW & -0.105 & PK:JAV & 0.033 & PK:PSC & -0.002 & & \\
\hline Non-TOM & & -0.056 & & 0.018 & & -0.008 & & \\
\hline
\end{tabular}

adaptive nature of equity returns. But, Urquhart and Hudson (2013) claim that the selections, choice, or size range of sub-samples is of a subjective nature. Thus the 5-year sub-sample contains an adequate set of observations to provide reliable and sufficient results to investigate the behavior of the TOM effect on how the abnormal effect behaves / performs over time. The above discussed empirical tests are employed on the 20 years (ranging from January 1996 to December 2015) equity returns of 107 firms (are available and in the possession of author which may be provided on demand.) listed on Pakistan Stock Exchange (PSX). The equity returns are calculated as under:-

$$
r_{t}=\left[\ln \left(P_{t}\right) / \ln \left(P_{t-1}\right)\right] \times 100
$$

For each firm's return at time $t, \ln \left(\mathrm{P}_{-} \mathrm{t}\right)$, denotes the natural logarithm of price of index at time $t$, and $\ln \left(\mathrm{P}_{-}(\mathrm{t}-1)\right)$ 
represents the natural logarithm of index price at $\mathrm{t}-1$. There are 5219 number of observations for each of 107 listed firms.

\section{Empirical Results}

Table 1 shows the results for TOM-effect during the period of full-sample (1996-2015) for all 107 firms. Standard ttest and K.W (non-parametric test) statistics are calculated to measure differences in mean. As compared to the normal days TOM days (Turn of the month) provide higher average returns in Pakistan. Both K.W and t-test support the presence of TOM-effect by demonstrating significant difference between mean returns on TOM and non-TOM days. Thus, we find statistically significant TOM-effect during full-sample period.

The table 2 elucidates average return on TOM anomaly days and non-TOM anomaly days for every firms individually through full sample period. Also, in $76.7 \%$ companies the average returns on TOM-days are greater than non TOMdays. Hence, based on average returns we find TOM anomaly in most of the sample firms during full-sample. Table 3 presents the behavior of TOM effect in full sample-period along with during sub samples-period. The findings reveal significant and positive TOM-effect in 34 companies [1] during full-sample period comprising 20 years (1996-2015); representing predictable, significant and positive returns around the turn of the month as the TOM effect claims. Meanwhile, 49 firms [2] generate positive but insignificant coefficient around the turn of the month in the full-sample. Thus, an overwhelming majority of companies under study display higher-positive mean returns around the turn of the month at PSX of Pakistan. While, 19 firms [3] generate negative-insignificant TOM effect and firms PK:DSM, PK:FZM, PK:GSM, PK:NON and PK:PNC produce significant and negative returns around TOM.

As for as sub-samples analysis is concerned TOM effect is insignificant in firms PK;ABB, PK;DAC, PK;DAW, PK;GTR, PK;HAE, PK;HUF, PK;JIN, PK;KWG, PK;PRE, PK;HPN, PK;SPP and PK;PBS in first sample-period (1996-2000), the behavior then reverses and becomes significant in next sub-sample. However the TOM effect again shows insignificant coefficients (market become efficient) in the next two consecutive sub-samples (2006-20010 and 2011-2015), thus supporting AMH (see Table 3, Panel A). Similarly, the firms PK;FSM, PK;GAI, PK;ASB, PK;MBK, PK;MRB, PK;NPK, PK;ORI, PK;SAP and PK;SER show independence of TOM effect in first two subsamples (1996-2000 and 2001-2005). The behavior of TOM effect reverses in third sub-sample (2006-2010) and becomes dependent which completely reverses and show independent behavior in the last sub-sample thus, consistent with AMH (see Table 3, Panel B).

Table 3: Results of GARCH $(1,1)$ model and k.w (Kruskal-Wallis) for TOM-Effect in sub and fullsample period for listed companies of PSX. Where TOM effect days are represented by" $\beta$ " while " $N$ " represents number of observations. The level of significance at $10 \%, 5 \% \& 1 \%$ is represented by $*$, ** \& *** respectively.

\begin{tabular}{|c|c|c|c|c|c|c|c|c|c|c|}
\hline $\mathrm{N}$ & Period & Firms & $\beta$ & K.w & Firms & $\beta$ & K.w & Firms & $\beta$ & K.w \\
\hline \multicolumn{11}{|c|}{ Panel-A } \\
\hline 5219 & Full-Sample & PK:ABB & $\begin{array}{l}0.1387 * * \\
(1.982)\end{array}$ & $2.6442 *$ & PK:HAE & $\begin{array}{l}0.2174 \\
(1.237)\end{array}$ & 2.1224 & PK:PRE & $\begin{array}{l}0.1426^{*} \\
(1.654)\end{array}$ & $4.4657 * *$ \\
\hline 1305 & $1996-2000$ & & $\begin{array}{l}0.0447 \\
(0.322) \\
\end{array}$ & 1.427 & & $\begin{array}{l}-0.1763 \\
(-0.022) \\
\end{array}$ & 0.816 & & $\begin{array}{l}-0.0864 \\
(-0.42) \\
\end{array}$ & 0.681 \\
\hline 1305 & $2001-2005$ & & $\begin{array}{l}0.3537 * * \\
(1.992)\end{array}$ & 0.09 & & $\begin{array}{l}-2.06 * * * \\
(-11.432)\end{array}$ & $3.695 *$ & & $\begin{array}{l}0.345 * * \\
(2.023)\end{array}$ & 1.559 \\
\hline 1304 & $2006-2010$ & & $\begin{array}{l}0.0755 \\
(0.681) \\
\end{array}$ & $2.8431 *$ & & $\begin{array}{l}0.1738 \\
(0.285) \\
\end{array}$ & $3.0118 *$ & & $\begin{array}{l}0.0833 \\
(0.683)\end{array}$ & 1.1173 \\
\hline 1305 & $2011-2015$ & & $\begin{array}{c}-0.0172 \\
(-0.168) \\
\end{array}$ & 0.0514 & & $\begin{array}{l}0.1461 \\
(0.523)\end{array}$ & 0.1662 & & $\begin{array}{l}0.1974 * \\
(1.644)\end{array}$ & $3.2081 *$ \\
\hline 5219 & Full-Sample & PK:DAC & $\begin{array}{l}0.0022 \\
(0.019) \\
\end{array}$ & 1.1623 & PK:HUF & $\begin{array}{l}-0.1405 \\
(-1.265) \\
\end{array}$ & 0.1503 & PK:HPN & $\begin{array}{l}0.084 \\
(0.883) \\
\end{array}$ & 0.3154 \\
\hline 1305 & 1996-2000 & & $\begin{array}{l}0.5712 * \\
(1.761)\end{array}$ & 1.531 & & $\begin{array}{l}0.1125 \\
(0.149) \\
\end{array}$ & 2.361 & & $\begin{array}{l}-0.0658 \\
(-0.264) \\
\end{array}$ & 0.012 \\
\hline 1305 & $2001-2005$ & & $\begin{array}{l}0.6541^{* * *} \\
(1.994)\end{array}$ & 0.004 & & $\begin{array}{l}0.8291 * * \\
(1.936)\end{array}$ & 0.386 & & $\begin{array}{l}0.2416^{* *} \\
(1.917)\end{array}$ & $5.819^{* *}$ \\
\hline
\end{tabular}




\begin{tabular}{|c|c|c|c|c|c|c|c|c|c|c|}
\hline 1304 & $2006-2010$ & & $\begin{array}{l}0.2611 \\
(0.867)\end{array}$ & 1.6347 & & $\begin{array}{l}-0.0849 \\
(-0.465)\end{array}$ & 0.0032 & & $\begin{array}{l}-0.0082 \\
(-0.04)\end{array}$ & 0.6777 \\
\hline 1305 & $2011-2015$ & & $\begin{array}{l}0.009 \\
(0.085)\end{array}$ & 0.2131 & & $\begin{array}{l}-0.182 \\
(-1.122) \\
\end{array}$ & 0.0101 & & $\begin{array}{l}-0.0492 \\
(-0.333) \\
\end{array}$ & 0.0512 \\
\hline 5219 & Full-Sample & PK:DAW & $\begin{array}{l}0.2433 * * \\
(2.532)\end{array}$ & $4.7778 * *$ & PK:JIN & $\begin{array}{l}0.1823 * \\
(1.813)\end{array}$ & 2.4049 & PK:SPP & $\begin{array}{l}0.0577 \\
(0.799)\end{array}$ & $4.2749 * *$ \\
\hline 1305 & $1996-2000$ & & $\begin{array}{l}0.0835 \\
(0.427)\end{array}$ & 0.102 & & $\begin{array}{l}-0.0024 \\
(-0.011)\end{array}$ & 0.004 & & $\begin{array}{l}0.2953 \\
(1.294)\end{array}$ & 0.001 \\
\hline 1305 & $2001-2005$ & & $\begin{array}{l}0.9065 * * * \\
(3.81)\end{array}$ & 1.174 & & $\begin{array}{l}0.399 * * \\
(2.001)\end{array}$ & 1.844 & & $\begin{array}{l}-1.346^{* * * *} \\
(-6.534)\end{array}$ & $3.706^{*}$ \\
\hline 1304 & $2006-2010$ & & $\begin{array}{l}0.017 \\
(0.118)\end{array}$ & $2.6305^{*}$ & & $\begin{array}{l}0.151 \\
(1.121)\end{array}$ & $2.7534 *$ & & $\begin{array}{l}0.0562 \\
(0.389)\end{array}$ & 0.829 \\
\hline 1305 & $2011-2015$ & & $\begin{array}{l}0.1774 \\
(1.165)\end{array}$ & 0.4084 & & $\begin{array}{l}0.0825 \\
(0.705)\end{array}$ & 0.0507 & & $\begin{array}{l}-0.0145 \\
(-0.112)\end{array}$ & 0.0148 \\
\hline 5219 & Full-Sample & PK:GTR & $\begin{array}{l}0.0499 \\
(0.62)\end{array}$ & 0.6213 & PK:KWG & $\begin{array}{l}-0.32 * * * \\
(-3.007)\end{array}$ & 0.6371 & PK:PBS & $\begin{array}{l}0.109 * * \\
(2.129)\end{array}$ & $11.2532 * * *$ \\
\hline 1305 & $1996-2000$ & & $\begin{array}{l}-0.1928 \\
(-1.064) \\
\end{array}$ & 0.152 & & $\begin{array}{l}-0.341 \\
(-1.213) \\
\end{array}$ & 0.211 & & $\begin{array}{l}-0.0305 \\
(-0.223) \\
\end{array}$ & 0.027 \\
\hline 1305 & $2001-2005$ & & $\begin{array}{l}0.3449 * * \\
(1.961)\end{array}$ & 1.732 & & $\begin{array}{l}-0.298 * * \\
(-2.02) \\
\end{array}$ & 0.795 & & $\begin{array}{l}0.3069 * * \\
(2.329)\end{array}$ & $7.279 * * *$ \\
\hline 1304 & $2006-2010$ & & $\begin{array}{l}-0.0789 \\
(-0.604) \\
\end{array}$ & 0.0855 & & $\begin{array}{l}-0.2457 \\
(-1.303) \\
\end{array}$ & 0.9391 & & $\begin{array}{l}-0.0184 \\
(-0.216) \\
\end{array}$ & 0.4889 \\
\hline 1305 & $2011-2015$ & & $\begin{array}{l}-0.0161 \\
(-0.103) \\
\end{array}$ & 0.359 & & $\begin{array}{l}-0.2559 \\
(-1.078) \\
\end{array}$ & 0.5294 & & $\begin{array}{l}0.0667 \\
(0.836) \\
\end{array}$ & $5.3797 * *$ \\
\hline \multicolumn{11}{|c|}{ Panel-B } \\
\hline 5219 & Full-Sample & PK:FSM & $\begin{array}{l}-0.0318 \\
(-0.237) \\
\end{array}$ & $3.2075^{*}$ & PK:MBK & $\begin{array}{l}0.1867 * * \\
(2.416) \\
\end{array}$ & $7.6248 * * *$ & PK:ORI & $\begin{array}{l}0.0927 \\
(1.095) \\
\end{array}$ & 1.4244 \\
\hline 1305 & $1996-2000$ & & $\begin{array}{l}-0.3876 \\
(-0.684) \\
\end{array}$ & 1.147 & & $\begin{array}{l}0.0181 \\
(0.088) \\
\end{array}$ & 0.264 & & $\begin{array}{l}-0.2731 \\
(-1.49) \\
\end{array}$ & 2.009 \\
\hline 1305 & $2001-2005$ & & $\begin{array}{l}-0.3016 \\
(-1.226)\end{array}$ & 2.173 & & $\begin{array}{l}0.2051 \\
(1.077)\end{array}$ & 0.666 & & $\begin{array}{l}0.1199 \\
(0.453)\end{array}$ & 0.085 \\
\hline 1304 & $2006-2010$ & & $\begin{array}{l}0.033 * * * \\
(8.126)\end{array}$ & 0.2053 & & $\begin{array}{l}0.3238^{* *} \\
(1.901)\end{array}$ & $5.4174 * *$ & & $\begin{array}{l}0.4067 * * * \\
(2.751)\end{array}$ & $3.2382 *$ \\
\hline 1305 & $2011-2015$ & & $\begin{array}{l}-0.0113 \\
(-0.086)\end{array}$ & 0.3568 & & $\begin{array}{l}0.1684 * \\
(1.769)\end{array}$ & 2.0708 & & $\begin{array}{l}0.069 \\
(0.431)\end{array}$ & 1.5471 \\
\hline 5219 & Full-Sample & PK:GAI & $\begin{array}{l}0.254 * \\
(1.931)\end{array}$ & 0.5372 & PK:MRB & $\begin{array}{l}-0.0573 \\
(-0.709)\end{array}$ & 0.1959 & PK:SAP & $\begin{array}{l}0.2227 * * \\
(2.472)\end{array}$ & 0.5838 \\
\hline 1305 & $1996-2000$ & & $\begin{array}{l}0.1322 \\
(0.149) \\
\end{array}$ & 0.018 & & $\begin{array}{l}-0.3395 \\
(-1.53) \\
\end{array}$ & $5.417 * *$ & & $\begin{array}{l}0.1864 \\
(1.023) \\
\end{array}$ & 0.367 \\
\hline 1305 & $2001-2005$ & & $\begin{array}{l}0.1802 \\
(0.873) \\
\end{array}$ & 0.425 & & $\begin{array}{l}-0.0219 \\
(-0.118) \\
\end{array}$ & 0.888 & & $\begin{array}{l}-0.3368^{*} \\
(-1.656) \\
\end{array}$ & $3.78 *$ \\
\hline 1304 & $2006-2010$ & & $\begin{array}{l}0.3364 * * \\
(1.915) \\
\end{array}$ & $4.9792 * *$ & & $\begin{array}{l}0.2939^{* *} \\
(1.909)\end{array}$ & 1.6513 & & $\begin{array}{l}0.6815 * * \\
(1.903) \\
\end{array}$ & $8.4408 * * *$ \\
\hline 1305 & $2011-2015$ & & $\begin{array}{l}-0.0952 \\
(-0.974) \\
\end{array}$ & $5.5136 * *$ & & $\begin{array}{l}-0.1272 \\
(-0.914) \\
\end{array}$ & 0.0321 & & $\begin{array}{l}0.0452 \\
(0.439) \\
\end{array}$ & 0.5781 \\
\hline 5219 & Full-Sample & PK:ASB & $\begin{array}{l}0.2692 \\
(0.974) \\
\end{array}$ & 0.8388 & PK:NPK & $\begin{array}{l}0.0209 \\
(0.321)\end{array}$ & 0.6002 & PK:SER & $\begin{array}{l}0.0752 \\
(0.602)\end{array}$ & 0.0321 \\
\hline 1305 & $1996-2000$ & & $\begin{array}{l}0.1181 \\
(0.248)\end{array}$ & 1.381 & & $\begin{array}{l}-0.0028 \\
(-0.023) \\
\end{array}$ & 1.383 & & $\begin{array}{l}-0.1246 \\
(-0.469) \\
\end{array}$ & 0.174 \\
\hline 1305 & $2001-2005$ & & $\begin{array}{l}0.1504 \\
(0.22)\end{array}$ & 0.733 & & $\begin{array}{l}0.0343 \\
(0.191)\end{array}$ & 2.237 & & $\begin{array}{l}-0.1132 \\
(-0.151)\end{array}$ & 0.253 \\
\hline 1304 & $2006-2010$ & & $\begin{array}{l}0.8352 * * \\
(1.984)\end{array}$ & $4.3817 * *$ & & $\begin{array}{l}0.2415^{* *} \\
(2.098)\end{array}$ & 0.7123 & & $\begin{array}{l}0.4441^{* *} \\
(1.982)\end{array}$ & $3.8345^{* *}$ \\
\hline 1305 & $2011-2015$ & & $\begin{array}{l}0.3357 \\
(0.931) \\
\end{array}$ & 0.7846 & & $\begin{array}{l}-0.0671 \\
(-0.605) \\
\end{array}$ & 0.0107 & & $\begin{array}{l}-0.1422 \\
(-1.09) \\
\end{array}$ & 0.1798 \\
\hline
\end{tabular}

The TOM effect in firms PK;AGT, PK;ATR, PK;BAP, PK;CTC, PK;DDH, PK;DEG, PK;FEC, PK;MET, PK;HUB, PK;KIE, PK;KNR, PK;MLC, PK;LDP, PK;PSO, PK;PSC, PK;TLM, PK;PTC, PK;PCT, PK;SNG and PK;SUI remain insignificant (independent) in first three subsamples (from years 19996-2010) and revert, predictable and moving towards dependency (market inefficiency) in last sub-sample (2011-2015) supporting AMH (see Table 4). Contrary to this behavior, firms PK;CAL, PK;CSA, PK;GLT, PK;GRY, PK;GUL, PK;GSM, PK;IMO and PK;RMP (see Table 5, Panel A) have significant TOM effect in first sub-sample (1996-2000) and produce insignificant coefficient in three consecutive sub-samples (2001-2015) and consistent with AMH. Also, firms PK;ADI, PK;FZM, PK;PAC and PA:POF support AMH (see Table 5, Panel B). 
Table 4: Results of GARCH $(1,1)$ model and k.w (Kruskal-Wallis) for TOM-Effect in sub and full-sample period for listed companies of PSX. Where TOM effect days are represented by" $\beta$ " while " $N$ " represents number of observations. The level of significance at $10 \%, 5 \% \& 1 \%$ is represented by $* * * \& * * *$ respectively.

\begin{tabular}{|c|c|c|c|c|c|c|c|c|c|}
\hline Period & Firms & $\beta$ & $K . w$ & Firms & $\beta$ & K.w & Firms & $\beta$ & K.w \\
\hline Full-Sample & PK:AGT & $\begin{array}{l}0.0885 \\
(1.241) \\
\end{array}$ & 1.7902 & PK:MET & $\begin{array}{l}0.1861 * * * \\
(2.674)\end{array}$ & $3.8617 * *$ & PK:PCT & $\begin{array}{l}0.3605 * * * \\
(3.442)\end{array}$ & $11.8177 * * *$ \\
\hline 1996-2000 & & $\begin{array}{l}0.012 \\
(0.054)\end{array}$ & 0.914 & & $\begin{array}{l}0.0803 \\
(0.438) \\
\end{array}$ & 0.172 & & $\begin{array}{l}0.1567 \\
(0.486) \\
\end{array}$ & 1.03 \\
\hline $2001-2005$ & & $\begin{array}{l}-0.0186 \\
(-0.121) \\
\end{array}$ & 0.674 & & $\begin{array}{l}0.185 \\
(1.281) \\
\end{array}$ & 0 & & $\begin{array}{l}0.1393 \\
(0.489) \\
\end{array}$ & 1.28 \\
\hline $2006-2010$ & & $\begin{array}{l}0.017 \\
(0.193)\end{array}$ & 0.1347 & & $\begin{array}{l}-0.0104 \\
(-0.077)\end{array}$ & 0.5359 & & $\begin{array}{l}0.1401 \\
(0.857)\end{array}$ & 2.4228 \\
\hline $2011-2015$ & & $\begin{array}{l}0.2329 * * \\
(2.135)\end{array}$ & 0.171 & & $\begin{array}{l}0.3371 * * * \\
(3.127)\end{array}$ & $7.4937 * * *$ & & $\begin{array}{l}0.6553 \text { *** } \\
(4.209)\end{array}$ & $7.0409 * * *$ \\
\hline Full-Sample & PK:ATR & $\begin{array}{l}0.1066 \\
(1.359)\end{array}$ & $6.9514 * * *$ & PK:KIE & $\begin{array}{l}0.4882 * * * \\
(4.695)\end{array}$ & $14.711 * * *$ & PK:SNG & $\begin{array}{l}0.1729 * * \\
(2.081)\end{array}$ & $10.8337 * * *$ \\
\hline 1996-2000 & & $\begin{array}{l}-0.1198 \\
(-0.702) \\
\end{array}$ & 0.045 & & $\begin{array}{l}0.4185^{*} \\
(1.675) \\
\end{array}$ & 1.27 & & $\begin{array}{l}0.019 \\
(0.101)\end{array}$ & 1.035 \\
\hline $2001-2005$ & & $\begin{array}{l}0.2011 \\
(1.036)\end{array}$ & 1.406 & & $\begin{array}{l}0.8353 \text { *** } \\
(4.131)\end{array}$ & $4.516^{* *}$ & & $\begin{array}{l}0.2715 \\
(1.399)\end{array}$ & $4.426^{* *}$ \\
\hline $2006-2010$ & & $\begin{array}{l}0.101 \\
(0.451)\end{array}$ & 0.0255 & & $\begin{array}{l}0.1583 \\
(0.736)\end{array}$ & $3.1587 *$ & & $\begin{array}{l}-0.0033 \\
(-0.021)\end{array}$ & 0.0026 \\
\hline $2011-2015$ & & $\begin{array}{l}0.3169 * * * \\
(2.815)\end{array}$ & $5.1029 * *$ & & $\begin{array}{l}0.5562 * * * \\
(3.173)\end{array}$ & $3.2147 *$ & & $\begin{array}{l}0.4 * * * \\
(3.208)\end{array}$ & $8.6338 * * *$ \\
\hline Full-Sample & PK:BAP & $\begin{array}{l}0.0145 \\
(0.187)\end{array}$ & 1.0839 & PK:MLC & $\begin{array}{l}0.4528 * * * \\
(4.467)\end{array}$ & $10.1752 * * *$ & PK:SUI & $\begin{array}{l}0.174 * * \\
(2.129)\end{array}$ & $7.8119 * * *$ \\
\hline $1996-2000$ & & $\begin{array}{l}0.1545 \\
(1.224)\end{array}$ & 0.856 & & $\begin{array}{l}0.0981 \\
(0.385)\end{array}$ & 0.002 & & $\begin{array}{l}0.1747 \\
(0.968)\end{array}$ & 0.726 \\
\hline $2001-2005$ & & $\begin{array}{l}-0.0838 \\
(-0.525)\end{array}$ & 0.557 & & $\begin{array}{l}0.4072 \\
(1.591)\end{array}$ & 1.655 & & $\begin{array}{l}0.056 \\
(0.284)\end{array}$ & 2.469 \\
\hline $2006-2010$ & & $\begin{array}{l}-0.0451 \\
(-0.256) \\
\end{array}$ & 0.1115 & & $\begin{array}{l}0.3795 * \\
(1.647) \\
\end{array}$ & $7.1606 * * *$ & & $\begin{array}{l}0.1186 \\
(0.7)\end{array}$ & 0.3967 \\
\hline $2011-2015$ & & $\begin{array}{l}-0.001 * * * \\
(-3.925)\end{array}$ & 1.2526 & & $\begin{array}{l}0.5853 * * * \\
(4.473)\end{array}$ & 1.0816 & & $\begin{array}{l}0.2746^{* * *} \\
(2.055)\end{array}$ & $4.7863 * *$ \\
\hline Full-Sample & PK:CTC & $\begin{array}{l}0.2265^{* *} \\
(2.501)\end{array}$ & $11.8777 * * *$ & PK:PSO & $\begin{array}{l}0.1795 * * \\
(2.294)\end{array}$ & $8.1584 * * *$ & PK:HUB & $\begin{array}{l}0.2831 * * * \\
(4.196)\end{array}$ & $24.3059 * * *$ \\
\hline 1996-2000 & & $\begin{array}{l}0.0185 \\
(0.084)\end{array}$ & 0.546 & & $\begin{array}{l}-0.0157 \\
(-0.093)\end{array}$ & 0.147 & & $\begin{array}{l}0.24 \\
(1.36)\end{array}$ & $5.73 * *$ \\
\hline $2001-2005$ & & $\begin{array}{l}0.0486 \\
(0.229)\end{array}$ & 2.463 & & $\begin{array}{l}0.2125 \\
(1.554)\end{array}$ & 2.236 & & $\begin{array}{l}0.1924 \\
(1.289)\end{array}$ & $6.563 * * *$ \\
\hline $2006-2010$ & & $\begin{array}{l}0.1069 \\
(0.661)\end{array}$ & $6.5885 * * *$ & & $\begin{array}{l}0.1799 \\
(0.887) \\
\end{array}$ & 0.4927 & & $\begin{array}{l}0.1135 \\
(0.826) \\
\end{array}$ & $2.5815^{*}$ \\
\hline $2011-2015$ & & $\begin{array}{l}0.4372 * * * \\
(2.971)\end{array}$ & 1.2622 & & $\begin{array}{l}0.277 * * * \\
(3.269)\end{array}$ & $5.8868 * *$ & & $\begin{array}{l}0.3176^{* * * *} \\
(3.358)\end{array}$ & $4.6044 * *$ \\
\hline Full-Sample & PK:DDH & $\begin{array}{l}0.2182^{* * *} \\
(2.529)\end{array}$ & $14.1214 * * *$ & PK:PSC & $\begin{array}{l}-0.0388 \\
(-0.341)\end{array}$ & 0 & PK:LDP & $\begin{array}{l}0.0924 \\
(1.527)\end{array}$ & $3.552 *$ \\
\hline 1996-2000 & & $\begin{array}{l}-0.0195 \\
(-0.086)\end{array}$ & 0.29 & & $\begin{array}{l}0.2506 \\
(0.991)\end{array}$ & 0.535 & & $\begin{array}{l}-0.0824 \\
(-0.515)\end{array}$ & 0.003 \\
\hline $2001-2005$ & & $\begin{array}{l}0.2166 \\
(1.337)\end{array}$ & $2.578^{*}$ & & $\begin{array}{l}-0.142 \\
(-0.667)\end{array}$ & 0.335 & & $\begin{array}{l}-0.0118 \\
(-0.102)\end{array}$ & 0.796 \\
\hline $2006-2010$ & & $\begin{array}{l}0.1574 \\
(1.288)\end{array}$ & $5.2897 * *$ & & $\begin{array}{l}0.4571 \\
(1.46)\end{array}$ & 0.1941 & & $\begin{array}{l}0.0937 \\
(0.85)\end{array}$ & 1.0504 \\
\hline $2011-2015$ & & $\begin{array}{l}0.3793 * * * \\
(2.814)\end{array}$ & $7.086 * * *$ & & $\begin{array}{l}-0.2783 * * \\
(-2.043) \\
\end{array}$ & 0.1806 & & $\begin{array}{l}0.3065 * * * \\
(2.772)\end{array}$ & $4.6954 * *$ \\
\hline Full-Sample & PK:DEG & $\begin{array}{l}0.3147 * * * \\
(3.713)\end{array}$ & $8.8173 * * *$ & PK:TLM & $\begin{array}{l}0.265 * * * \\
(3.558)\end{array}$ & $16.5606^{* * *}$ & PK:KNR & $\begin{array}{l}0.1849 \\
(1.177) \\
\end{array}$ & $7.8623 * * *$ \\
\hline $1996-2000$ & & $\begin{array}{l}0.0461 \\
(0.198)\end{array}$ & 0.54 & & $\begin{array}{l}0.3846^{*} \\
(1.727) \\
\end{array}$ & $5.749 * *$ & & $\begin{array}{l}-0.8324^{*} \\
(-1.613)\end{array}$ & 2.256 \\
\hline $2001-2005$ & & $\begin{array}{l}0.1983 \\
(0.99)\end{array}$ & 0.248 & & $\begin{array}{l}0.0997 \\
(0.798)\end{array}$ & 1.561 & & $\begin{array}{l}0.3999 \\
(1.341)\end{array}$ & 2.338 \\
\hline
\end{tabular}




\begin{tabular}{|c|c|c|c|c|c|c|c|c|}
\hline $2006-2010$ & & $\begin{array}{l}0.1398 \\
(0.792) \\
\end{array}$ & 1.8912 & & $\begin{array}{l}0.003 \\
(0.022)\end{array}$ & 0.4957 & $\begin{array}{l}0.2846 \\
(1.446)\end{array}$ & $5.8293 * *$ \\
\hline $2011-2015$ & & $\begin{array}{l}0.5168 \text { *** } \\
(4.565) \\
\end{array}$ & $9.6967 * * *$ & & $\begin{array}{l}0.4468 * * * \\
(3.767) \\
\end{array}$ & $9.947 * * *$ & $\begin{array}{l}0.5259 * * * \\
(2.693)\end{array}$ & 1.4519 \\
\hline Full-Sample & PK:FEC & $\begin{array}{l}0.077 \\
(0.74) \\
\end{array}$ & 1.3354 & PK:PTC & $\begin{array}{l}0.1644 * \\
(1.695)\end{array}$ & $4.0282 * *$ & & \\
\hline $1996-2000$ & & $\begin{array}{l}-0.2339 \\
(-0.983) \\
\end{array}$ & 0.231 & & $\begin{array}{l}0.0146 \\
(0.058) \\
\end{array}$ & 0.089 & & \\
\hline $2001-2005$ & & $\begin{array}{l}0.2473 \\
(1.004)\end{array}$ & 0.393 & & $\begin{array}{l}0.133 \\
(0.547)\end{array}$ & 0.21 & & \\
\hline $2006-2010$ & & $\begin{array}{l}0.0657 \\
(0.378) \\
\end{array}$ & 0.0052 & & $\begin{array}{l}-0.095 \\
(-0.628) \\
\end{array}$ & 0.1086 & & \\
\hline $2011-2015$ & & $\begin{array}{l}0.5063 * * * \\
(2.699)\end{array}$ & 1.6 & & $\begin{array}{l}0.3455 * * \\
(2.408) \\
\end{array}$ & $7.2405 * * *$ & & \\
\hline
\end{tabular}

Table 5: Results of GARCH (1,1) model and k.w (Kruskal-Wallis) for TOM-Effect in sub and fullsample period for listed companies of PSX. Where TOM effect days are represented by" $\beta$ " while "N" represents number of observations. The level of significance at $10 \%, 5 \% \& 1 \%$ is represented by $*$, $* * \& * * *$ respectively.

\begin{tabular}{|c|c|c|c|c|c|c|c|c|c|}
\hline Period & Firms & $\beta$ & $K . w$ & Firms & $\beta$ & $K . w$ & Firms & $\beta$ & $K . w$ \\
\hline \multicolumn{7}{|c|}{ Panel-A } & \multicolumn{3}{|c|}{ Panel-B } \\
\hline Full-Sample & PK:CAL & $\begin{array}{l}0.5881 * * \\
(2.227)\end{array}$ & 1.2168 & PK:GUL & $\begin{array}{l}0.0622 \\
(0.797)\end{array}$ & 1.7375 & PK:ADI & $\begin{array}{l}0.2023 * * \\
(2.388)\end{array}$ & $7.6042 * * *$ \\
\hline 1996-2000 & & $\begin{array}{l}1.9316^{* * * *} \\
(11.941)\end{array}$ & $2.973^{*}$ & & $\begin{array}{l}0.1607 * * \\
(2.42)\end{array}$ & 0.024 & & $\begin{array}{l}0.0667 \\
(0.318)\end{array}$ & 1.641 \\
\hline 2001-2005 & & $\begin{array}{l}0.5917 \\
(0.622)\end{array}$ & 0.055 & & $\begin{array}{l}-0.1634 \\
(-0.963)\end{array}$ & 0.451 & & $\begin{array}{l}0.3686^{* * *} \\
(2.089)\end{array}$ & $4.474 * *$ \\
\hline 2006-2010 & & $\begin{array}{l}0.2049 \\
(0.469)\end{array}$ & 0.0006 & & $\begin{array}{l}0.0639 \\
(0.263)\end{array}$ & 1.0908 & & $\begin{array}{l}0.1629 \\
(0.829)\end{array}$ & 0.9466 \\
\hline 2011-2015 & & $\begin{array}{l}0.9636 \\
(1.482)\end{array}$ & 0.0826 & & $\begin{array}{l}0.0823 \\
(0.618)\end{array}$ & 0.3221 & & $\begin{array}{l}0.2298 * * \\
(2.001)\end{array}$ & 1.1182 \\
\hline Full-Sample & PK:CSA & $\begin{array}{l}0.0598 \\
(0.667) \\
\end{array}$ & 2.008 & PK:GSM & $\begin{array}{l}-0.1724^{*} \\
(-1.917)\end{array}$ & 0.9381 & PK:FZM & $\begin{array}{l}-0.515 * * * \\
(-5.431)\end{array}$ & 0.1053 \\
\hline $1996-2000$ & & $\begin{array}{l}-0.3362 * * \\
(-1.98)\end{array}$ & 1.447 & & $\begin{array}{l}-0.68 * * * \\
(-4.456)\end{array}$ & 0.33 & & $\begin{array}{l}-0.995 * * * \\
(-2.595)\end{array}$ & 0.013 \\
\hline 2001-2005 & & $\begin{array}{l}0.2068 \\
(0.885)\end{array}$ & 0.786 & & $\begin{array}{l}0.0939 \\
(0.334)\end{array}$ & 0.646 & & $\begin{array}{l}0.0275 \\
(0.164)\end{array}$ & 0.154 \\
\hline $2006-2010$ & & $\begin{array}{l}0.1148 \\
(0.508)\end{array}$ & 0.1857 & & $\begin{array}{l}0.1595 \\
(0.768)\end{array}$ & 0.2943 & & $\begin{array}{l}1.269 * * * \\
(3379.564)\end{array}$ & 2.3522 \\
\hline 2011-2015 & & $\begin{array}{l}0.1439 \\
(1.088)\end{array}$ & 1.118 & & $\begin{array}{l}0.3913 \\
(1.342)\end{array}$ & 0.778 & & $\begin{array}{l}0.0426 \\
(0.322)\end{array}$ & 0.612 \\
\hline Full-Sample & PK:GLT & $\begin{array}{l}0.1386^{* *} \\
(2.542)\end{array}$ & $3.0801 *$ & PK:IMO & $\begin{array}{l}0.0661 \\
(0.917)\end{array}$ & 0.0892 & PK:PAC & $\begin{array}{l}0.1461 \\
(1.577)\end{array}$ & $3.0257^{*}$ \\
\hline 1996-2000 & & $\begin{array}{l}0.2451 * * \\
(2.296)\end{array}$ & $2.751^{*}$ & & $\begin{array}{l}0.4363 * * \\
(2.009)\end{array}$ & $5.054 * *$ & & $\begin{array}{l}-0.494 * * * \\
(-6.798)\end{array}$ & 0.018 \\
\hline 2001-2005 & & $\begin{array}{l}0.0961 \\
(0.783)\end{array}$ & 1.711 & & $\begin{array}{l}-0.0259 \\
(-0.163)\end{array}$ & 0.609 & & $\begin{array}{l}0.1347 \\
(1.202)\end{array}$ & 0.637 \\
\hline $2006-2010$ & & $\begin{array}{l}0.0163 \\
(0.183) \\
\end{array}$ & 0.0113 & & $\begin{array}{l}-0.0963 \\
(-0.7) \\
\end{array}$ & 2.0904 & & $\begin{array}{l}0.0399 \\
(0.23)\end{array}$ & 0.0469 \\
\hline 2011-2015 & & $\begin{array}{l}0.1973 * \\
(1.814)\end{array}$ & 0.6611 & & $\begin{array}{l}0.1951 * \\
(1.883)\end{array}$ & 1.593 & & $\begin{array}{l}0.3999 * * * \\
(3.571)\end{array}$ & $7.2322 * * *$ \\
\hline Full-Sample & PK:GRY & $\begin{array}{l}0.0415 \\
(0.363)\end{array}$ & 0.0266 & PK:RMP & $\begin{array}{l}-0.0002 \\
(-0.004)\end{array}$ & 0.5628 & PK:POF & $\begin{array}{l}0.2837 * * * \\
(4.751)\end{array}$ & $11.6213 * * *$ \\
\hline $1996-2000$ & & $\begin{array}{l}0.219 * * * \\
(3.106)\end{array}$ & 0.494 & & $\begin{array}{l}-0.31 * * * \\
(-173.46)\end{array}$ & 0.256 & & $\begin{array}{l}0.2647 * * \\
(2.361)\end{array}$ & 0.137 \\
\hline $2001-2005$ & & $\begin{array}{l}-0.0991 \\
(-0.295) \\
\end{array}$ & 1.318 & & $\begin{array}{l}0.1169 \\
(1.315) \\
\end{array}$ & 0.968 & & $\begin{array}{l}0.0618 \\
(0.392) \\
\end{array}$ & $4.701 * *$ \\
\hline $2006-2010$ & & $\begin{array}{l}0.3308 \\
(0.988)\end{array}$ & 0.0034 & & $\begin{array}{l}-0.0167 \\
(-0.171)\end{array}$ & 0.4491 & & $\begin{array}{l}0.1299 \\
(0.848)\end{array}$ & 0.6314 \\
\hline 2011-2015 & & $\begin{array}{l}-0.1394 \\
(-0.723) \\
\end{array}$ & 1.8486 & & $\begin{array}{l}-0.0081 \\
(-0.057) \\
\end{array}$ & 1.1083 & & $\begin{array}{l}0.2607 * * * \\
(3.177) \\
\end{array}$ & $3.9506^{* *}$ \\
\hline
\end{tabular}


Contrary to this behavior, firms PK;ACB, PK;ERO, PK;FAU, PK;KIE, PK;NAR and PK;CCB show insignificant TOM effect in the first sub-sample (1996-2000), while the behavior of TOM effect reverses in the next sub-sample and becomes dependent in (2001-2005). The sub-sample 2006-2010 reveals a complete reversal of TOM effect which prevails in sub-period (2001-2005), then again turns to significant TOM effect in the last sub-sample (2011-2015) supporting AMH (see Table 6). Therefore, 59 firms (55\% of the sample size) show behavior of TOM effect consistent with AMH, means TOM effect fluctuate over time. While TOM effect in firms [4] remains independent and does not evolve over time as all the sub-samples produce insignificant coefficient.

Table 6: Results of GARCH $(1,1)$ model and k.w (Kruskal-Wallis) for TOM-Effect in sub and fullsample period for listed companies of PSX. Where TOM effect days are represented by" $\beta$ " while "N" represents number of observations. The level of significance at $10 \%, 5 \% \& 1 \%$ is represented by *, ** \& *** respectively.

\begin{tabular}{|c|c|c|c|c|c|c|c|c|c|}
\hline Period & Firms & $\beta$ & K.w & Firms & $\beta$ & K.w & Firms & $\beta$ & K.w \\
\hline Full-Sample & PK:ACB & $\begin{array}{l}0.2549 * * * \\
(3.829)\end{array}$ & $18.6673 * * *$ & PK:FAU & $\begin{array}{l}0.1725 * * * \\
(3.794)\end{array}$ & $17.7898 * * *$ & PK:CCB & $\begin{array}{l}0.1166 \\
(0.855) \\
\end{array}$ & $3.6365^{*}$ \\
\hline 1996-2000 & & $\begin{array}{l}0.2417 \\
(1.421)\end{array}$ & $7.355^{* * *}$ & & $\begin{array}{l}0.155 \\
(1.009)\end{array}$ & 0.773 & & $\begin{array}{l}-0.2479 \\
(-0.817)\end{array}$ & 0.523 \\
\hline 2001-2005 & & $\begin{array}{l}0.3765 \text { *** } \\
(2.621)\end{array}$ & 2.2 & & $\begin{array}{l}0.3547 * * * \\
(2.665)\end{array}$ & $8.007 * * *$ & & $\begin{array}{l}0.6777 \text { ** } \\
(2.474)\end{array}$ & $4.688 * *$ \\
\hline $2006-2010$ & & $\begin{array}{l}0.1534 \\
(1.303)\end{array}$ & $4.5662 * *$ & & $\begin{array}{l}0.1535^{*} \\
(1.676)\end{array}$ & $5.718 * *$ & & $\begin{array}{l}0.0465 \\
(0.197)\end{array}$ & 1.084 \\
\hline 2011-2015 & & $\begin{array}{l}0.2709 * * \\
(2.166)\end{array}$ & $3.9097 * *$ & & $\begin{array}{l}0.1397 * * \\
(2.2)\end{array}$ & $3.8678 * *$ & & $\begin{array}{l}1.248 * * * \\
(3719.402)\end{array}$ & 0.0805 \\
\hline Full-Sample & PK:ERO & $\begin{array}{l}0.2621 * * * \\
(3.954)\end{array}$ & $15.1403 * * *$ & PK:KIE & $\begin{array}{l}0.4882 * * * \\
(4.695)\end{array}$ & $14.711 * * *$ & PK:NAR & $\begin{array}{l}0.2606 * * * \\
(2.955)\end{array}$ & $9.8936 * * *$ \\
\hline 1996-2000 & & $\begin{array}{l}0.074 \\
(0.541) \\
\end{array}$ & 0.44 & & $\begin{array}{l}0.4185^{*} \\
(1.675) \\
\end{array}$ & 1.27 & & $\begin{array}{l}-0.0471 \\
(-0.326) \\
\end{array}$ & 0.08 \\
\hline $2001-2005$ & & $\begin{array}{l}0.3171 * * * \\
(2.753)\end{array}$ & 2.516 & & $\begin{array}{l}0.8353 * * * \\
(4.131)\end{array}$ & $4.516 * *$ & & $\begin{array}{l}0.4038^{* *} \\
(2.346) \\
\end{array}$ & $2.78 *$ \\
\hline $2006-2010$ & & $\begin{array}{l}0.2405^{*} \\
(1.668)\end{array}$ & $4.3549 * *$ & & $\begin{array}{l}0.1583 \\
(0.736) \\
\end{array}$ & $3.1587 *$ & & $\begin{array}{l}0.0917 \\
(0.372) \\
\end{array}$ & 0.119 \\
\hline 2011-2015 & & $\begin{array}{l}0.3907 * * * \\
(3.233)\end{array}$ & $7.2605 * * *$ & & $\begin{array}{l}0.5562 * * * \\
(3.173)\end{array}$ & $3.2147 *$ & & $\begin{array}{l}0.4663 * * * \\
(4.51)\end{array}$ & $6.6453 * * *$ \\
\hline
\end{tabular}

\section{Conclusion}

Although, the modern research favors the fact that TOM effect anomaly is diminished or even reversed with the passage of time. But the capacious published work exhibits the presence and acceptance of TOM effect in all stock exchanges of world. The study explore the time varying nature of TOM-effect to elucidate whether or not TOM-effect is used to capture the excess returns. The study finds $77 \%$ firms' display positive and higher average profits on TOM (turn of month) days, therefore, exhibits the existence of TOM anomaly via application of GARCH model and K.W test over the sample period (1996-2015). Finally, analysis of sub sample reveals TOM-effect (Appendix 1) in 59 companies swings between the eras of market efficiency/predictability and market inefficiency/no-predictability or vice versa, while TOM effect do not swing in 48 companies in sub sample periods. Therefore, it is concluded that behavior TOM effect at firm level is best elucidated by AMH than traditional/classical EMH at PSX.

In summary, it is inferred that firms listed at PSX exhibit time varying behavior with the utilization of sub-sample analysis. This symbol of time variation in the behavior of turn of month effect (TOM) is supporting and consistent with the implications of $\mathrm{AMH}$ while contrasting to classical EMH. We recommend further researchers to use a long time period sub-sample to explore the idea of AMH and propose that the method used in this study could be helpful and adapted to inspect other anomalous market effect or calendar effects in emerged and emerging stock markets around the globe. 
[1] PK:ABB, PK:ADI, PK:ACB, PK:BKP, PK:CAL, PK:CTC, PK:DDH, PK:DAW, PK:DEG, PK:ERO, PK:FAU, PK:GLT, PK:HAB, PK:MET, PK:HSM, PK:HUB, PK:ICI, PK:JIN, PK:KIE, PK:KWG, PK:MLC, PK:MBK, PK:NAR, PK:NHT, PK:POF, PK:PRE, PK:PSO, PK:TLM, PK:PTC, PK:PCT, PK:SAP, PK:PBS, PK:SNG and PK:SUI.

[2] PK:AGT, PK:ATR, PK:BAP, PK:CPB, PK:CSA, PK:CTX, PK:CYA, PK:DAC, PK:DAE, PK:DAN, PK:DKT, PK:DMT, PK:DES, PK:FEC, PK:GAI, PK:GTR, PK:GWC, PK:GRY, PK:GUL, PK:HAE, PK:HPM, PK:IMO, PK:INI, PK:ASB, PK:JAV, PK:KNR, PK:LDP, PK:NPK, PK:NAT, PK:ORI, PK:PAC, PK:PET, PK:PSM, PK:PAL, PK:PNS, PK:PSM, PK:LAK, PK:POC, PK:RUP, PK:STM, PK:CCB, PK:SAN, PK:HPN, PK:SPP, PK:SEA, PK:SER, PK:SHJ, PK:SON and PK:TRP.

[3] PK:AGR, PK:ATH, PK:BHA, PK:BOC, PK:DEW, PK:ETU, PK:FSM, PK:NAK, PK:HUF, PK:KRM, PK:MIR, PK:MRB, PK:PEN, PK:PSC, PK:RMP, PK:SHA, K:SCM, PK:SHK and PK:SIT.

[4] PK;AGR, PK;ATR, PK;BKP, PK;BHA, PK;BOC, PK;CPB, PK;CTX, PK;CYA, PK;DAE, PK;DAN, PK;DKT, PK;DMT, PK;DES, PK;DSM, PK;DEW, PK;ETU, PK;NAK, PK;GWC, PK;HAB, PK;HSM, PK;HPM, PK;INI, PK;JAV, PK;KRM, PK;MIR, PK;NAT,PK;NHT, PK;NON, PK;PET, PK;PSM, PK;PNC, PK;PEN, PK;PAL, PK;PNS, PK;PSM, PK;LAK, PK;POC, PK;RUP, PK;STM, PK;SAN, PK;SEA, PK;SHA, PK;SCM, PK;SHJ, PK;SHK, PK;SIT,PK;SON and PK;TRP.

\section{Reference}

Abdul, H., Hung-Chun, L., \& Fakhar-un-Nisa. (2011). Testing the Weak Form Efficiency of Pakistani stock Market (2000-2010). International journal of Economics and Financial Issues, 1(4), 153-162.

Agrawal, A., \& Tandon , K. (1994). Anomalies or Illusion? Evidence from Stock Markets in Eighteen Countries. Journal of international Money and Finance, 13, 83-106. doi:10.1016/0261-5606(94)90026-4

Ariel, R. A. (1987). A Monthly Effect in Stock Returns. Journal of Financial Economics, 18(1), 161-174. doi: $10.1016 / 0304-405 \times(87) 90066-3$

Bollerslev, T. (1986). Generalized Autoregressive Conditional Heteroskedasticity. Journal of Econometrics, 307-327. doi:10.1.1.468.2892

Cadsby, C. B., \& Ratner, M. (1992). Turn of the Month and Pre-Holiday Effects on Stock Returns: Some International Evidence. Journal of Banking And Finance, 16(3), 497-509. doi: 0378-4266(92)90041-W

Campbell, J. Y., Lo, A. W., \& Mackinlay, A. C. (1997). The Econometrics of Financial Markets. (N. Princeton, Ed.) Princeton University Press.

Camptom, W. S., Johson, D. T., \& Kunkel, R. A. (2006). The Turn of the Month Effect in Real estate Investment. Managerial Finance, 32(12), 969-980. doi: 10.1108/03074350610710463

Chen, H., \& Chua, A. (2011). The Turn of Month Anomli in the Age of ETFs: A Reexamination of Return Enhancement Strategies. Journal of Financial Planning, 62-67.

Ehsan, L. (2012). Stock Market Psychology in Pakistan. International Journal of Governance, 2(5), 1-8.

Engle, R. (2001). GARCH 101: The use of ARCH/GARCH Models in Applied Econometrics. Journal of Economic Perspectives, 15(4), 157-168. doi: 10.1257/jep.15.4.157

Fama, F. E. (1970). Efficient Capital Markets: A Review of Theory and Empirical Work. Journal of Finance, 25(2), 383-417. doi: $10.2307 / 2325486$

Grossman, S. J., \& Stiglitz, J. E. (1980). On The Impossibility of Informationally Efficient Markets. The American Economic Review, 393-408. doi: 10.7916/D8765R99 
Halari, A. (2013). An Analysis of Monthly Calendar Anomalies in the Pakistani Stock Market: A Study of the Gregorian and Islamic Calendars. University of Dundee: $\mathrm{PhD}$. Thesis.

Hashmi, M. A. (2014). January Effect in Pakistan: A Time Series Analysis. Market Forces, IX(1), 37-44.

Hensel, C. R., \& Ziemba, W. T. (1996). Investment Results from Exploiting Turn-of-the-Month Effects. Journal of Portfolio Management, 22(3), 43-45. doi: 10.3905/jpm.1996.409556

Iqbal, M. S., Kouser, R., \& Azeem, M. (2013). Conventional and Islamic Anomalies in Karachi Stock Exchange. Science International, 25(4), 999-1007.

Lakonishok, J., \& Smidt, S. (1988). Are seasonal Anomalies Real? A Ninety Year Perspective. The Review of Financial Studies, 1(4), 403-425.

Lo, A. W. (2004). The Adaptive Market Hypothesis. Journal of Portfolio Management, 30, 15-29. doi: $10.3905 / j p m .2004 .442611$

McConnell, J. J., \& Xu, W. (2008). Equity Returns at the Turn of the Month. Financial Analysts Journal, 62(2), 4964. doi: $10.2469 /$ faj.v64.n2.11

Nisar, S., \& Hanif, M. (2012). Testing Weak form of Market Hypothesis: Empirical Evidence from South Asia. World Applied Sciences Journal, 17(4), 414-427.

Rabbani, S., Kamal, N., \& Salim, M. (2013). Testing the Weak Form Efficiency of the Stock Market: Pakistan as an Emerging Economy. Journal of Basic and Applied Scientific Research, 3(4), 136-142.

Riaz, T., Hassan, A., \& Nadim, M. (n.d). Market Efficiency in its Weak Form; Evidence from Karachi Stock Exchange of Pakistan. The Journal of Commerce, 4(4), 9-17.

Sanaullah, A., Shah, S. M., Athar, M., \& Ali, E. (2012). How Behavior Aspect Affect Market Efficiency-Evidence from KSE 100 Index. Global Journal of Management and Business Research, 10.

Sania, \& Rizwan, M. (2014). Testing Weak Form Efficiency of Capital Markets: A Case of Pakistan. International Journal of Research Studies in Management, 3(1), 65-73.

Shahid, M. N., \& Mehmood, Z. (2015). Calendar Anomalies in Stock Market: A Case of KSE 100 Index. International Journal of African and Asian Studies, 7, 16-23.

Shahid, M. N., \& Sattar, A. (2017). Behavior of Calendar Anomalies, Market Conditions and Adaptive Market Hypothesis: Evidence from Pakistan Stock Exchange. Pakistan Journal of Commerce and Social Sciences, 11(2), 471-504.

Shahid, M. N., Jabeen, S., Sattar, A., \& Ateeq, A. (2018). Behavior of Bitcoin Returns and Adaptive Market Hypothesis (AMH). Asia Pacific Journal of Emerging Markets, 2(2), 113.

Silva, P. (2010). Calendar Anomalies in The Portguese Stock Market. Investment Analysis Journal, 71, 37-47. doi; $10.1080 / 10293523.2010 .11082518$

Urquhart, A. (2013). An Empirical Analysis of the Adaptive Market Hypothesis and Investor Sentiment in Extreme Circumstances. PhD Thesis.

Urquhart, A., \& Hudson, R. (2013). Efficient or Adaptive Markets? Evidence from Major Stock Markets using very Long-Run Historic Data. International Review of Financial Analysis, 28, 130-142. doi: $\underline{10.1016 / j . i r f a .2013 .03 .005}$

Urquhart, A., \& McGroarty, F. (2014). Calendar Effects, Market Conditions and the Adaptive Market Hypothesis: Evidence from Long-Run Data. International Review of Financial Analysis, 35, 154-166. doi: 10.1016/j.irfa.2014.08.003 
Wong, W. K., Agarwal, A., \& Wong, N.-T. (2006). The Disappearing Calendar Anomalies in the Singapore Stock Market. The Lahore Journal of Economics, 11(2), 123-139.

Zafar, N., Urooj, S. F., Chughtai, S., \& Amjad, S. (2012). Calendar Anomalies: Case of Karachi Stock Exchange. African Journal of Business Administration, 6(24), 7261-7271. 